\title{
Telehealth in Australian cardiology: insight into factors predicting the use of telephone versus video during the COVID-19 pandemic
}

\author{
Stephanie J. Rowe (D), Elizabeth D. Paratz (D), Louise Fahy, David L. Prior and Andrew I. Maclsaac \\ Department of Cardiology, St Vincent's Hospital Melbourne, Melbourne, Victoria, Australia
}

\section{Key words}

telemedicine, telehealth, COVID-19, ambulatory

care, cardiology.

Correspondence

Stephanie J. Rowe, Department of Cardiology, St

Vincent's Hospital Melbourne, 41 Victoria

Parade, Fitzroy, Melbourne, Vic. 3065, Australia.

Email: stephanie.rowe@svha.org.au

Received 2 October 2020; accepted

26 June 2021

\begin{abstract}
Background: The use of telehealth has increased dramatically in Australia in 2020 as a pragmatic response to the COVID-19 pandemic; however, differences between telehealth modalities have not been established.

Aim: To identify characteristics contributing to choosing telephone (TP) versus video consultation (VC) and assess patient outcomes between telehealth modalities.

Methods: We conducted an observational study of cardiology outpatients at a tertiary hospital with appointments from 17 March 2020 to 12 August 2020. Demographic variables and appointment modality were compared between each group. Outcomes assessed were mortality, emergency department (ED) presentations and cross over between appointment modalities.

Results: There were 1754 telemedicine encounters with 1188 patients seen by TP and 327 patients by VC. Consulting volume increased from previous years. Cardiac mortality was low $(0.3 \%)$. There were no differences in mortality or ED presentations between telehealth modalities. Patients choosing TP over VC were older $(P<0.001)$, more likely to be female $(P=0.005)$, non-English-speaking $(P=0.041)$, living in metropolitan Melbourne $(P<0.0001)$, undertaking a first appointment $(P=0.002)$ and seeing particular cardiologists $(P<0.001)$. VC patients were more likely to have early review $(P=0.015)$, and this was likely to be TP $(P<0.0001)$. TP patients were more likely to follow up in person $(P<0.0001)$.

Conclusion: During COVID-19, we increased consultation volumes without adverse patient outcomes. We identified factors influencing the choice of telemedicine modality which did not translate into differences in mortality or ED presentations. Telemedicine is a growing platform with an important role of facilitating access to healthcare for diverse patient groups.
\end{abstract}

\section{Introduction}

The COVID-19 pandemic has dramatically altered the delivery of ambulatory patient care worldwide. Concerns about the risk of viral transmission during visits to medical facilities have driven a decline in face-to-face (FTF) consultations. Alternative strategies to maintain patient access to outpatient specialist services have been implemented. ${ }^{1}$ Telemedicine has been widely promoted around the world as a safe alternative to FTF

Funding: None.

Conflict of interest: None. interactions. ${ }^{2}$ It is an evolving field encompassing the delivery of healthcare services at a geographical distance by healthcare professionals using information and communication technologies. ${ }^{3}$ The most common modalities used are voice-only telephone (TP) and video-based conferences (video consultation (VC)).

Previously in Australia, telemedicine resources were predominantly used to assist patients living in a rural or remote setting to access medical care, with Medicare only reimbursing these restricted scenarios. ${ }^{4}$ In response to the COVID-19 pandemic, on 13 March 2020, the Australian Government opened access to telemedicine to a wider group of patients (vulnerable patients or those in 
self-isolation) under the Medicare Benefits Schedule. ${ }^{5}$ By 29 March 2020, eligibility was broadened to all Australian patients to maximise access to remote healthcare during the pandemic. ${ }^{6}$

With the rapid expansion of telemedicine services in Australia, there has been increasing discussion around the effectiveness of telemedicine compared to FTF consultations, as well as challenges experienced by patients and healthcare providers. Systematic reviews have previously found that telemedicine can be used as an adjunct, or a suitable replacement to usual care in patients with chronic conditions. ${ }^{7-9}$ However, the effectiveness of different telemedicine modalities, such as TP versus VC, as well as the driving factors behind patient and doctor preference for a particular modality have not thoroughly been investigated. Objective data regarding patient safety outcomes for both modalities in the Australian pandemic context are also needed.

The aim of this study was to assess the use of telemedicine in the cardiology ambulatory setting at our institution during the COVID-19 pandemic. We evaluated the usage of telephone versus audio-visual telehealth (TP vs VC) and identified patient- and doctor-related factors that may contribute to the choice of telemedicine modality. We assessed key clinical patient outcomes (mortality, emergency department (ED) presentations and likelihood of patients choosing telehealth at subsequent appointments) and whether these differed according to the initial choice of telehealth modality.

\section{Methods}

Data were collected from 17 March 2020 to 12 August 2020 from all cardiology clinics conducted at St Vincent's Hospital Melbourne. St Vincent's Hospital Melbourne is an 880-bed general hospital in Melbourne, Victoria, Australia, with a tertiary cardiology inpatient and outpatient service. This time period reflects the initial transition to telemedicine during 'stay at home' lockdown restrictions in Melbourne due to COVID-19. During this time frame, the hospital executive directed that all outpatient services should be provided in telehealth format (either telephone or video) unless medically unavoidable. All patients were notified that their appointment was changed to a VC as a default. Telephone consultation was performed if either patient or treating cardiologist elected for this mode. FTF appointments were only permitted if clinically necessary.

Using electronic medical records and Medicare billing codes, we obtained details of 2169 appointments during the specified time frame. Patients were included if they completed an appointment using telemedicine. Appointment modality was classified as telephone-only (TP) or VC (utilising the hospital-provided video call platform) or FTF in-person consultations.

Demographics obtained were age, gender, preferred language, rural status, date of appointment, whether the patient and next of kin had the same address (as a surrogate marker of likelihood of the patient living alone), private insurance status, type of appointment (defined as initial vs review, and simple vs complex) and treating cardiologist. Whether a patient had been referred to clinic as a result of an ED presentation or recent admission in the 6 weeks prior was also established.

The primary outcome assessed was mortality (both all-cause and cardiac) during the study period as this is the most important clinical outcome for patient care. Secondary outcomes assessed were ED presentations within the study period (both all-cause and cardiac-related) and the number of patients who crossed over between appointment modalities at subsequent appointments. ED presentations were assessed in order to encompass patients who required clinical assessment and management in the ED as well as those who may have required subsequent admission to hospital.

Exclusion criteria were incarcerated patients $(n=47)$ as telehealth appointments for this group were almost universal prior to the COVID pandemic, and patients who had multidisciplinary appointments involving simultaneous consultations with multiple specialties $(n=141)$.

This project was reviewed and approved as a Quality Assurance project (QA 20078) by the Ethics Committee of St Vincent's Hospital Melbourne, and complies with the principles of the National Statement on the Ethical Conduct of Human Research (NHMRC; 2007).

\section{Statistical analysis}

Patient demographics and outcomes were compared according to whether they conducted their appointment using TP or VC. Categorical data are presented as numbers and percentages, with significance assessed using a chi-square test. Continuous variables are presented as medians and interquartile ranges, and compared using a Mann-Whitney test. Multiple regression was performed on outcome variables to adjust for baseline differences identified in the demographic analysis. $P<0.05$ was considered statistically significant. All statistics were computed using STATA statistical software 


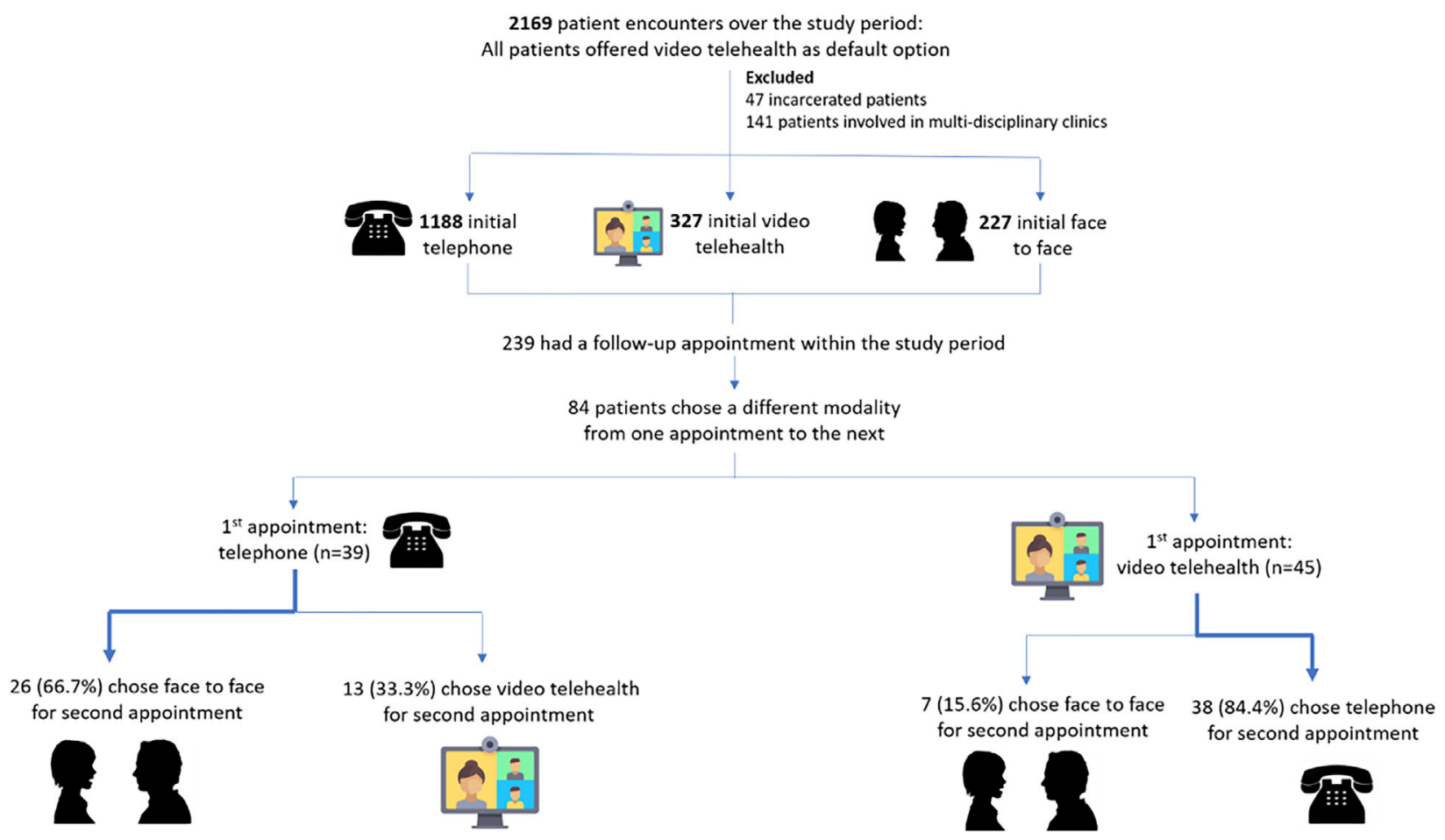

Figure 1 Patient encounters.

packaging (STATA v14.2; StataCorp LLC, College Station, TX, USA).

\section{Results}

The study period comprised 148 days with 112235 patient-days of clinical follow up (307.5 patient-years). During the study period, there were 2169 patient encounters through St Vincent's Outpatient Cardiology Clinics, with 179 patients excluded as per pre-specified study criteria. This patient volume reflected a $21.5 \%$ increase in consultations from the year prior. Reasons for this increase may be due to improved compliance to outpatient appointments due to no travel requirement, or shorter appointments allowing for increased capacity due to lack of physical examination. There were 1754 telemedicine encounters (Fig. 1) in 1515 patients (485 initial outpatient consultations, 1269 review consultations and 239 patients had follow-up appointments within the study period), where $0.9 \%$ of telemedicine encounters were new referrals from ED presentations and $1.7 \%$ of appointments were as a result of a recent cardiology-related admission. There were 227 FTF encounters ( $10 \%$ of encounters).

Overall, $1188(78.4 \%)$ patients were seen by TP and $327(21.6 \%)$ patients by VC appointment. There were significant baseline differences (Table 1) with regard to age $(P<0.001$; Fig. 2$)$, gender $(P=0.005)$, preferred first language $(P=0.041)$, rural status $(P<0.0001)$, first appointment status $(P=0.002)$ and consulting cardiologist $(P<0.001)$. Patients having a TP appointment were older, more likely to be female, lived in metropolitan Melbourne, did not have English as a first language and were more likely to be attending a review appointment. The variation in type of telemedicine consultation was significant when analysed by the treating cardiologist.

There were no statistical differences between groups with regard to next of kin living at the same residence, interpreter use or private health insurance status.

With regard to the primary outcome, there were no differences between groups in terms of either all-cause or cardiac mortality. There were five cases of cardiac mortality; three of these cases were deemed 'expected deaths' on case review. Secondary outcome analysis showed no differences between groups in terms of presentations to the ED, including both all-cause and cardiac-related reasons for presentation.

Patients assessed by videoconferencing appointments were more likely to have a follow-up appointment than those seen using telephone $(P=0.015)$. Of those who had a follow-up appointment (Table 2, Fig. 1), patients initially using the video modality were more likely to choose an alternative modality for the next appointment $(57.0$ vs $19.9 \%, P<0.0001)$, and 
Table 1 Baseline demographics of patients choosing telephone versus telehealth

\begin{tabular}{|c|c|c|c|}
\hline & Telephone & Video telehealth & Significance $(P)$ \\
\hline Overall number & $1188(78.4 \%)$ & $327(21.6 \%)$ & \\
\hline Age (years) & $67(54-76)$ & $61(46-71)$ & $<0.0001$ \\
\hline Male gender (\%) & $670(56.4 \%)$ & $213(65.1 \%)$ & 0.005 \\
\hline English preferred language (\%) & $1028(86.5 \%)$ & $294(89.9 \%)$ & 0.041 \\
\hline $\begin{array}{l}\text { Interpreter used in those } \\
\text { with non-English-speaking } \\
\text { background (\%) }\end{array}$ & $18(11.3 \%)$ & $4(12.1 \%)$ & 0.696 \\
\hline $\begin{array}{l}\text { Next of kin does not live at } \\
\text { the same residence (\%) }\end{array}$ & $362(30.5 \%)$ & $78(23.9 \%)$ & 0.640 \\
\hline Private health insurance (\%) & $193(16.2 \%)$ & $68(20.8 \%)$ & 0.054 \\
\hline Rural patient & $135(11.4 \%)$ & $61(18.7 \%)$ & $<0.0001$ \\
\hline Initial appointment (\%) & $324(27.3 \%)$ & $118(36.1 \%)$ & 0.002 \\
\hline Complex appointment (\%) & $31(2.6 \%)$ & $10(3.1 \%)$ & 0.650 \\
\hline
\end{tabular}

$\dagger$ Fifteen cardiologists were allocated into tertiles of five cardiologists each according to proportion of telephone/telehealth consults seen. $\mathrm{IQR}$, interquartile range.

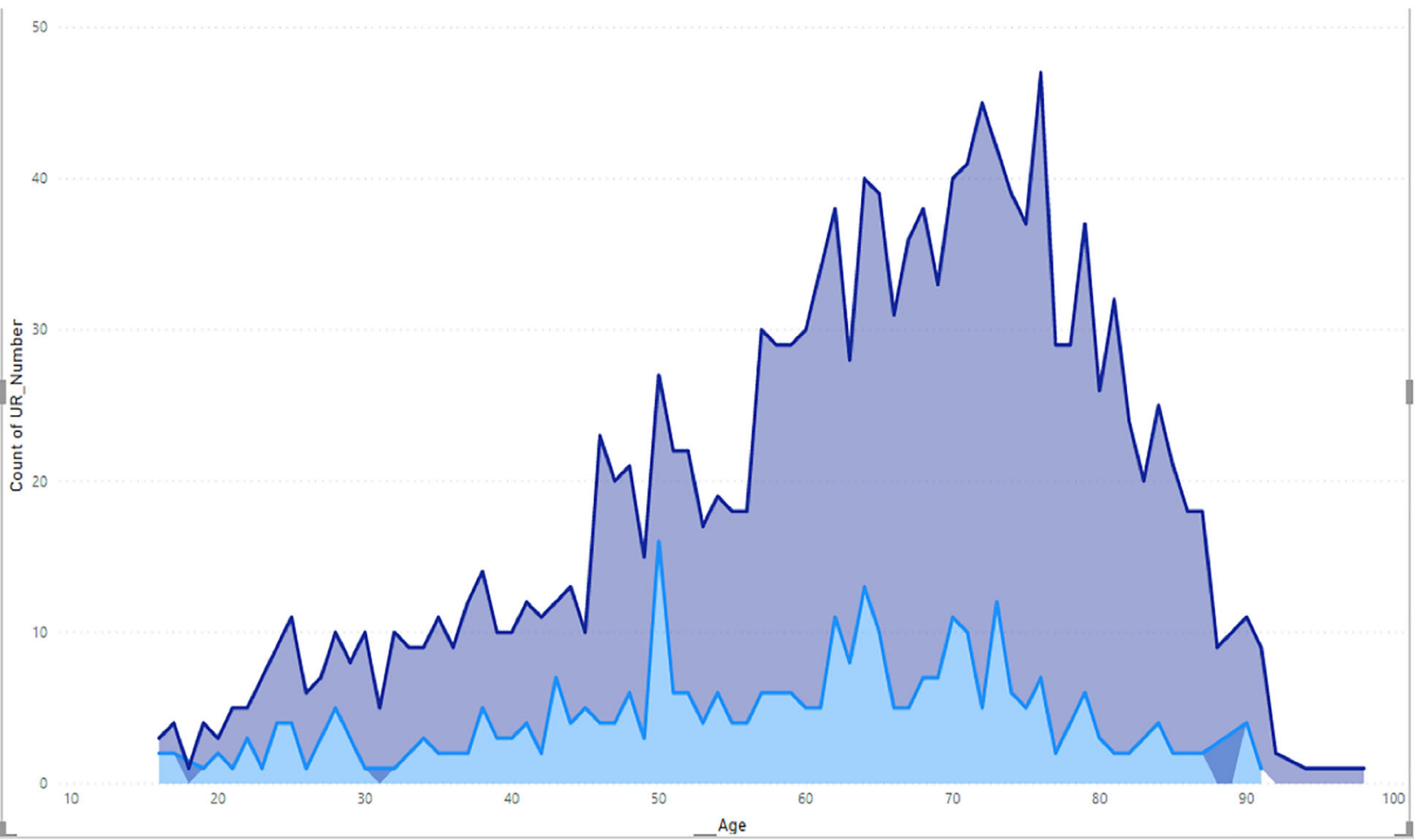

Figure 2 Age ranges for audio-visual telehealth (light blue) versus telephone (dark blue) patients.

this modality was more likely to be an alternative telemedicine mode ( 84.4 vs $33.3 \%, P<0.0001)$. Patients who used the TP modality initially were more likely to choose FTF appointments as follow up (66.7 vs 15.6\%, $P<0.0001)$. All of these findings remained significant after adjusting for baseline differences identified in demographic analysis.

\section{Discussion}

The COVID-19 pandemic has changed the face of ambulatory patient care in Australia. A key consideration in hospitals' logistical pandemic planning was minimising all non-essential visits to hospital facilities, including ambulatory patients. Remote delivery of healthcare to 
Table 2 Outcomes for patients choosing telephone versus telehealth

\begin{tabular}{|c|c|c|c|c|}
\hline & Telephone & Video telehealth & Significance $(P)$ & Adjusted significance $(P) \dagger$ \\
\hline Number & $1188(78.4 \%)$ & $327(21.6 \%)$ & & \\
\hline \multicolumn{5}{|l|}{ Subsequent appointments } \\
\hline Follow-up appointment within the study time period & 196 (16.5\%) & 79 (24.2\%) & 0.002 & 0.015 \\
\hline Chose alternative modality for next appointment & 39 (19.9\%) & $45(57.0 \%)$ & $<0.0001$ & $<0.0001$ \\
\hline Chose alternative telehealth mode (TP- > VC or VC- > TP) $\S$ & $13(33.3 \%)$ & $38(84.4 \%)$ & $<0.0001$ & $<0.0001$ \\
\hline Chose face to face for next appointment ${ }^{a}$ & $26(66.7 \%)$ & $7(15.6 \%)$ & $<0.0001$ & $<0.0001$ \\
\hline \multicolumn{5}{|l|}{ ED presentations } \\
\hline ED presentation during the study period & $118(9.9 \%)$ & 18 (5.5\%) & 0.013 & 0.165 \\
\hline Cardiac reason for ED presentation during the study periodII & $25(21.2 \%)$ & $3(16.7 \%)$ & 0.659 & 0.511 \\
\hline \multicolumn{5}{|l|}{ Mortality } \\
\hline All-cause mortality during the study period & $12(1.0 \%)$ & $2(0.6 \%)$ & 0.505 & 0.806 \\
\hline Cardiac mortality during study period $\dagger \dagger$ & $4(0.3 \%)$ & $1(0.3 \%)$ & 0.931 & 0.759 \\
\hline
\end{tabular}

$\dagger$ Multiple regression performed to adjust for baseline differences of age, gender, English as first language, rural status, initial appointment status and cardiologist seen.

$\ddagger$ Of those who had a follow-up appointment within the time period.

$\S$ of those who changed modality within the study period.

IIDenominator used for these variables is patients who had an ED presentation.

$\uparrow \dagger$ These deaths included four deaths of patients who utilised telephone consult, and one death of a patient who utilised VC. These cases were adjudicated by a panel of cardiologists. Three out of five deaths were deemed to be expected given the patient history and prognosis.

ED, emergency department; TP, telephone consultation; VC, video consultation.

cardiovascular patients has been shown to be vital in order to support usual care. ${ }^{10}$ The large body of literature published since the pandemic suggests that proactive use of telemedicine may also play a role in reducing the long-term effects of experiencing lockdowns and social isolation. ${ }^{10}$

During this study period, the total number of cardiology consultations performed increased compared with previous years, suggesting that access to specialist care was able to be maintained throughout this challenging time. While maintaining access to cardiac specialist care, 1754 in-person visits were averted and completed as telemedicine appointments, likely resulting in public health benefits by reducing risk of viral transmission. FTF visits accounted for only $10 \%$ of all consultations. Both patient- and doctor-related factors were identified that drove the choice of telehealth modality, but these did not translate into differences regarding mortality or ED usage. This suggests that both TP and VC are safe ways of providing ongoing care for cardiology patients during the pandemic.

Given the increasing use of telemedicine and the significant baseline differences in patients choosing TP and VC, it is important to appreciate this may impact patient care. We used a primary end-point of mortality as being the most important patient outcome (including both allcause and cardiac mortality), with secondary consideration of ED presentations. Overall, cardiac mortality in this cohort was low $(0.3 \%)$ and did not reflect differences in telehealth modality usage. The secondary end- point of ED presentations encompassed both hospital admissions and patients discharged from the ED. During the study period, there was encouragement to manage patients on an outpatient basis where able, both to preserve hospital bed capacity in a pandemic and minimise patients' risk of viral transmission. ED presentations rather than admitted inpatient episodes were therefore chosen as the most holistic end-point capturing symptomatic patient presentations.

Our findings are concordant with a recent systematic review examining differences in telehealth outcomes, which demonstrated that although VC may offer improved diagnostic accuracy and reduced medication errors, patient outcomes including mortality are equivalent to TP. ${ }^{11}$

Certain aspects of FTF consultation are lost with telemedicine. A variety of technological tools have been created to assist with such differences, but these are yet to be widely implemented. ${ }^{10,12}$ Key issues raised are the lack of physical examination, lack of non-verbal cues and the dependence on technology and adequate Internet connection that can be challenging. With TP use, there are also perceived challenges that come with not having visual rapport or connection with a patient and the impact this may have on a consultation. ${ }^{13}$ However, for healthcare professionals, telemedicine can provide the opportunity for more flexible work practices, ease pressure on administration staff as well as assist patients and doctors alike in reducing travel times and breaking down geographical barriers. 
In our study, the strongest predictors of choosing TP over VC were older age and living in a metropolitan location. Other significant determinants included female gender, not speaking English as a first language and if the appointment was a review visit. Recently, concerns have been raised regarding the potential for telemedicine to exacerbate inequities with more vulnerable patients, including older age and non-English-speaking patients, being less likely to utilise telemedicine and specifically less likely to utilise video encounters. ${ }^{14}$ Our results support the notion that there are baseline differences between patients choosing different modalities. Older age as a factor in choosing telephone consultation is in keeping with the known relationship between age and digital literacy. ${ }^{15}$ Previous experience with using Internet and other technology is a key factor in confidence and technology usage in later years. ${ }^{15}$ Telephone is therefore an important option for older Australians when it comes to telemedicine and was non-inferior with regard to patient outcomes.

Prior to the pandemic, telemedicine was predominantly focussed on supporting remote Australian patients. Telemedicine programmes in rural Australia can be vital in reducing waiting times for tests and travel time for patients, ${ }^{16}$ and there has been increasing uptake in telemedicine use over recent years. ${ }^{17}$ We found that a greater proportion of patients in rural settings used VC than metropolitan location patients, which may be related to rural patients being more likely to have experienced audio-visual telehealth before and therefore being more prepared for increased reliance on telemedicine.

Although we identified several patient-related factors involved in modality choice, we also found that there was significant variation between cardiologists in terms of which modality they were likely to utilise for an encounter. This implies that it is not only patient-related factors that play a role in the choice of modality. Doctors, as well as patients, have varying confidence with technology and they may be the driving factor in encouraging FTF review if they have clinical concerns regarding a patient. Further investigation is required to assess potential reasons behind this.

In the ambulatory setting, the patient-doctor relationship builds over repeated visits, and follow-up appointments become important in terms of re-assessing symptoms and refining differential diagnoses. Patients were less likely to have follow up within the study time period if the encounter had been using TP as opposed to VC. Patients who had follow-up appointments within the study period changed modalities in very different ways. $84.4 \%$ of VC patients who changed modality transitioned to TP for subsequent appointments. However, $69.2 \%$ of TP patients who changed modality transitioned to FTF review. These differences are likely attributable to a combination of patient and doctor preference, such as difficulty using audio-visual technology, which may lead to avoidance of this modality for follow up. Those who initially utilised telephone may have been lacking the infrastructure or skill to utilise VC initially, therefore they would be less likely to transition to $\mathrm{VC}$ in the future. In contrast, those who initially used VC had the skills to do so, so the reasoning behind their transition to TP is less clear and may be driven by patient or doctor preference. Although it has been suggested that patient and doctor experience is similar with FTF and $\mathrm{VC},{ }^{11,18,19}$ there is less evidence on experience with TP consultation. The ways in which people changed between modalities suggest potential inequity between the telemedicine modalities in terms of patient and doctor experience.

\section{Limitations}

There are multiple limitations to this study. With regard to patient outcomes, our study only includes ED presentations and mortality that occurred through our institution unless the hospital had been notified by other means. In addition to this, our study follow-up time is relatively short to assess for mortality as an end point, particularly given that cardiovascular disease can take years to progress. Finally, further qualitative investigation is warranted to develop a better understanding into why individual patients or doctors chose a specific modality, or what experiences they had with each modality. Patient and doctor perception of the adequacy and usefulness of telemedicine could also be addressed in future research.

\section{Conclusion}

The COVID-19 pandemic has led to an increased use of telemedicine. Our study demonstrates that during the COVID-19 pandemic we were able to maintain consultation volumes with very low levels of cardiac mortality and ED usage. Patient- and doctor-related factors were associated with choice of telehealth modality, but did not translate into different outcomes. Beyond COVID-19, telemedicine is a growing platform which has an important role of facilitating adequate access to healthcare for diverse patient groups. 


\section{References}

1 Zaman S, Macisaac AI, Jennings GL, Schlaich MP, Inglis SC, Arnold R et al. Cardiovascular disease and COVID-19: Australian and New Zealand consensus statement. Med J Aust 2020; 213: 182-7.

2 Monaghesh E, Hajizadeh A. The role of telehealth during COVID-19 outbreak: a systematic review based on current evidence. BMC Public Health 2020; 20: 1193.

3 Sood S, Mbarika V, Jugoo S, Dookhy R, Doarn CR, Prakash N et al. What is telemedicine? A collection of 104 peerreviewed perspectives and theoretical underpinnings. Telemed J E Health 2007; 13: $573-90$

4 Bradford NK, Caffery LJ, Smith AC. Telehealth services in rural and remote Australia: a systematic review of models of care and factors influencing success and sustainability. Rural Remote Health 2016; 16: 3808

5 Prime Minister of Australia. \$2.4 billion health plan to fight COVID-19 [media release]. cited 2020 Mar 11. Available from URL: https://www.pm.gov.au/ media/24-billion-health-plan-fightcovid-19

6 Prime Minister of Australia. \$1.1 billion to support more mental health, Medicare and domestic violence services [media release]. 2020 Mar 29. Available from URL: https://www.pm.gov.au/ media/1 1-billion-support-more-mentalhealth-medicare-and-domesticviolence-services- 0
7 Inglis SC, Clark RA, Dierckx R, PrietoMerino D, Cleland JG. Structured telephone support or non-invasive telemonitoring for patients with heart failure. Cochrane Database Syst Rev 2015; CD007228.

8 Lee SWH, Chan CKY, Chua SS, Chaiyakunapruk N. Comparative effectiveness of telemedicine strategies on type 2 diabetes management: a systematic review and network metaanalysis. Sci Rep 2017; 7: 12680.

9 Kotb A, Cameron C, Hsieh S, Wells G. Comparative effectiveness of different forms of telemedicine for individuals with heart failure (HF): a systematic review and network meta-analysis. PLoS One 2015; 10: e0118681.

10 Neubeck L, Hansen T, Jaarsma T, Klompstra L, Gallagher R. Delivering healthcare remotely to cardiovascular patients during COVID-19. Eur J Cardiovasc Nurs 2020; 19: 486-94.

11 Rush KL, Howlett L, Munro A, Burton L. Videoconference compared to telephone in healthcare delivery: a systematic review. Int J Med Inform 2018; 118: 44-53.

12 Linz D, Pluymaekers N, Hendriks JM. TeleCheck-AF for COVID-19. Eur Heart J 2020; 41: 1954-5.

13 Parker J, Niranjan S, Sriram KB. A case of broken heart syndrome via the telephone: socially distant outpatient clinics in the COVID-19 pandemic. Intern Med J 2020; 50: 1429-31.

14 Eberly LA, Khatana SAM, Nathan AS, Snider C, Julien HM, Deleener ME et al.
Telemedicine outpatient cardiovascular care during the COVID-19 pandemic: bridging or opening the digital divide? Circulation 2020; 142: 510-2.

15 Office of the eSafety Commissioner, Australian Government Understanding the digital behaviours of older Australians: summary of national survey and qualitative research. 2018 [cited 2020 Sep 27]. Available from URL: https://www.esafety.gov.au/sites/ default/files/2019-08/Understandingdigital-behaviours-older-Australianssummary-report-2018.pdf

16 Scott AC, McDonald A, Roberts T, Martin C, Manns T, Webster M et al. Cardiovascular telemedicine program in rural Australia. N Engl J Med 2020; 383: 883-4.

17 Australian Government, Department of Health. Telehealth quarterly statistics update. 2016. Available from URL: http://www.mbsonline.gov.au/internet/ mbsonline/publishing.nsf/Content/ connectinghealthservices-factsheet-stats

18 Tates K, Antheunis ML, Kanters S, Nieboer TE, Gerritse MB. The effect of screen-to-screen versus face-to-face consultation on doctor-patient communication: an experimental study with simulated patients. J Med Internet Res 2017; 19: e421.

19 Donelan K, Barreto EA, Sossong S, Michael C, Estrada JJ, Cohen AB et al. Patient and clinician experiences with telehealth for patient follow-up care. Am J Manag Care 2019; 25: 40-4. 